\title{
8.4 M Deep Space Habitat- Medical Bay Concept Design and Human Factors Engineering Analysis
}

\author{
Mary Claire Mancl ${ }^{1}$ and Tanya C. Andrews ${ }^{2}$ \\ ${ }^{I}$ Student (Department of Mechanical Engineering; University of Wisconsin-Madison) \\ Madison, WI, USA \\ ${ }^{2}$ Human Factors Engineer (EV74 Human Factors Engineering; NASA Marshall \\ Spaceflight Center) Huntsville, AL, USA
}

\begin{abstract}
Maintaining crew health throughout long duration deep space missions presents significant new design challenges. Since communication with Earth will be limited and it is not possible to return in the event of a medical emergency it is critical that medical care on a deep space mission be as autonomous as possible. The goal of this project was to design and assess a medical system concept for the Deep Space Habitat Concept Demonstrator derived from the core stage of the Space Launch System. The Medical Bay was designed as a primary location to support the medical care of $4 \mathrm{crew}$ members on a 1000-day mission. It includes workspace, stowage, and direct access to all necessary medical equipment and supplies. A Human Factors Engineering Analysis was conducted to test ergonomic effectiveness and system requirement compliance. This initial design will continue to be used by the Human Factors Engineering Team and the Advanced Concepts Office at NASA's Marshall Spaceflight Center for further demonstration, analysis, and design conception for deep space travel.
\end{abstract}

\section{Introduction}

As NASA prepares to send humans further into space and for longer periods of time than ever before it becomes increasingly necessary to develop new efficient systems for keeping crew alive and healthy for the length of their mission. With the ultimate goal of sending humans to Mars, the Advanced Concepts Office (ACO) at the NASA Marshall Spaceflight Center (MSFC) has developed an 8.4 meter diameter Deep Space Habitat Concept (DSHC) to contain everything that is required to support four crew members on a 1000-day mission into deep space.

This habitat is derived from the core stage of the Space Launch System (SLS) and is sized to be the largest possible payload to be flown in SLS Configuration 1B (NASA, 2019). The Human Factors Engineering (HFE) team at MSFC is responsible for analyzing the safety, usability, and ergonomics of human interface equipment within this concept. They have constructed a full-sized Deep Space Habitat Concept Demonstrator (DSHCD), shown in Figure 1, to facilitate further development and to run HFE analyses on the concept.

On a mission to Mars one-way communication with Earth can take up to 22 minutes and it would not be possible to return to Earth for medical emergencies. Crew therefore must have direct access to all required equipment, supplies, and facilities to autonomously treat and prevent anticipated illness and injury in deep space. In collaboration with HFE and ACO the goal of this project was to develop medical system requirements and to design, mockup and assess a concept for a Medical Bay as a primary medical treatment location within the DSHC.

* This NASA project was made possible through support from the Wisconsin Space Grant Consortium. 

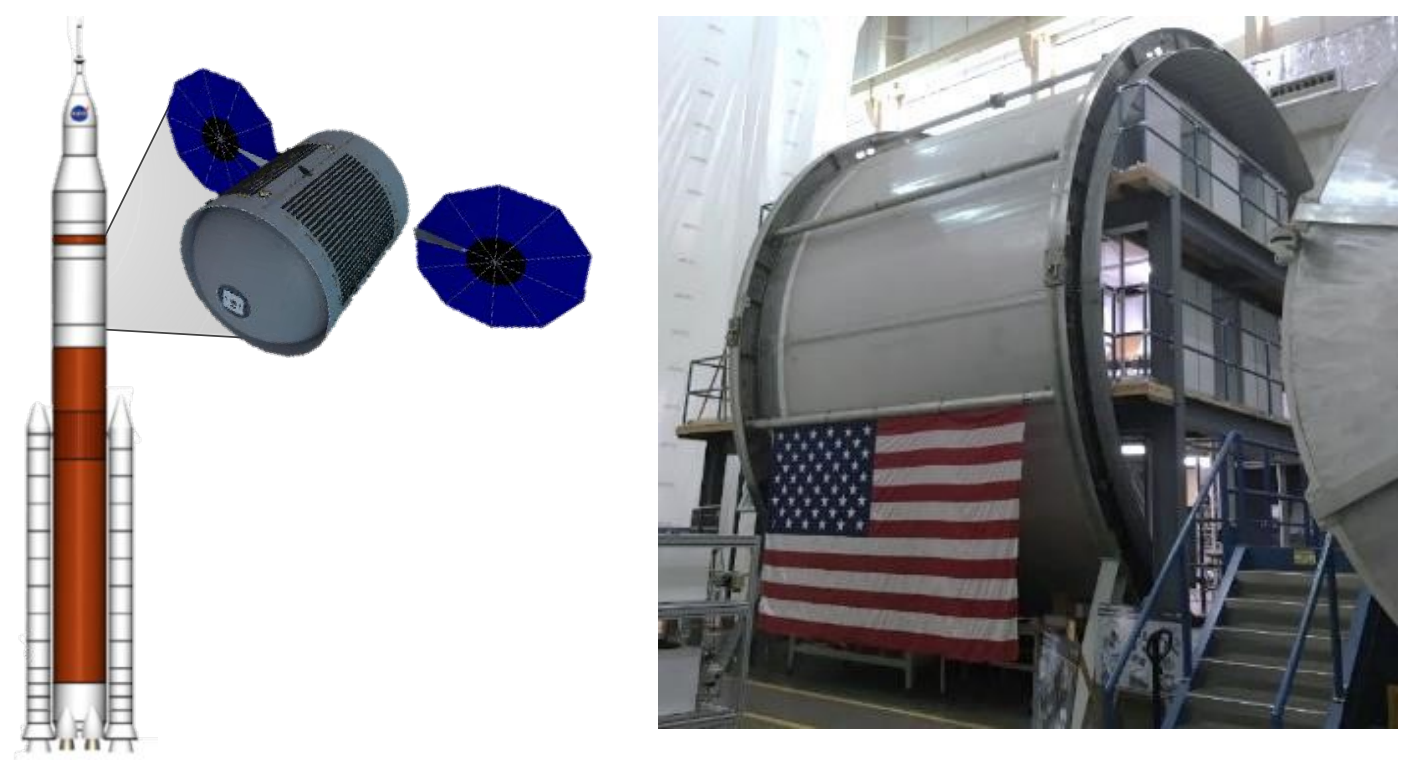

Figure 1: (Left) Space Launch System Configuration 1B that will fly the 8.4 M Deep Space Habitat as a payload (Right) 8.4 M Deep Space Habitat Concept Demonstrator at NASA's Marshall Spaceflight Center

\section{System Requirements Development}

System requirements were developed to indicate what capabilities must exist within the Medical System in the deep space habitat. The requirements were intended to facilitate the design of the Medical System concept and were not intended to be an exhaustive list of everything that must be considered when designing for flight. These requirements expand on NASA Human Factors, Habitability and Environmental Health standards, as well as common practice medical standards with specific reference to NASA-STD-3001 Vol 1 and 2 (NASA, 2015).

The Medical Bay was specified to be sized for the $1^{\text {st }}$ percentile female to the $99^{\text {th }}$ percentile male population based on the 1988 U.S. Army Anthropometry Survey database and was required to be contained within the volume allocation given by the ACO (US Army, 1988). Additional anthropometric constraints and space accommodations such as size, work volume, range of motion, and reach were also specified.

The Medical System was required to have the capability for providing the highest level of medical care specified in NASA-STD-3001 Vol 1. This includes capabilities such as first aid, clinical diagnostics, trauma care, dental care, autonomous advanced life support and ambulatory care, and basic surgical care (NASA, 2015). Specifications for crew safety, equipment accessibility, and orientation and labeling were also included. 


\section{Medical Bay Design}

The Medical Bay was designed to fulfill the Medical System Requirements and to be the primary location for medical care within the DPHC. Medical supplies were selected to provide the best possible care while minimizing volume and mass. The layout, shown in Figure 3, was designed in the 3D modeling software, Creo Parametric, with the intent to organize all medical supplies so treatment can be provided in the easiest and most efficient manner.
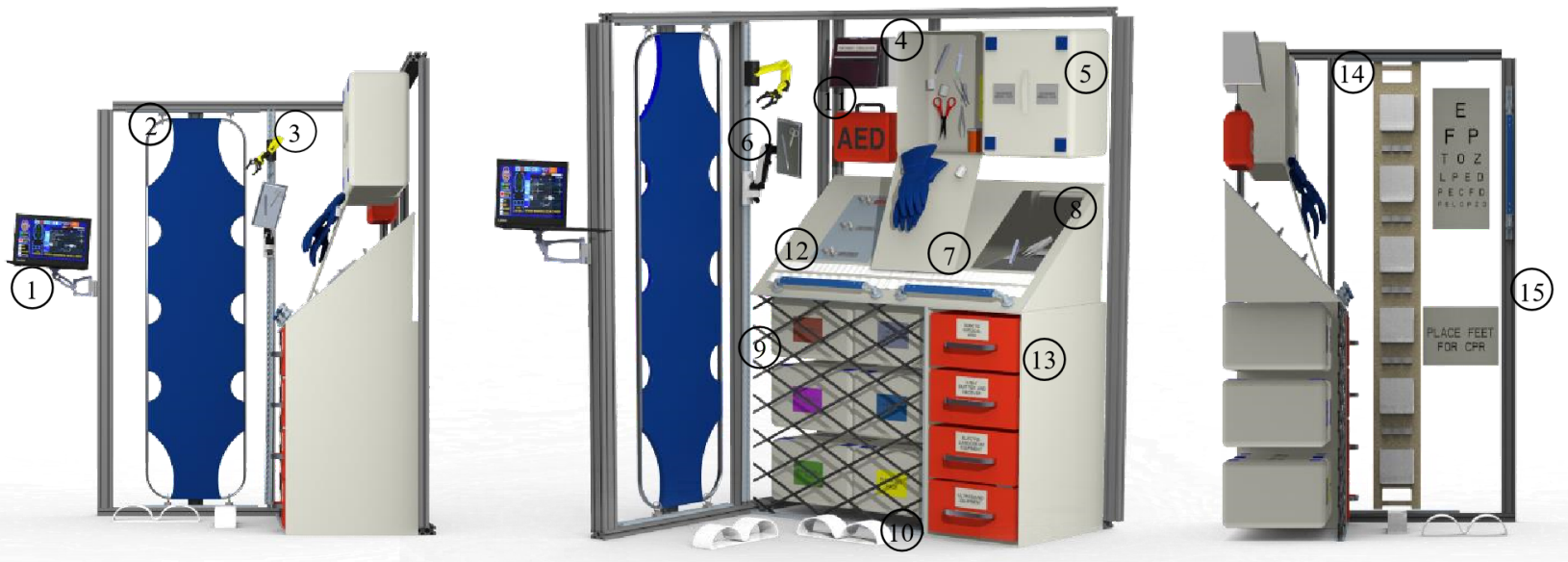

Medical Bay Design Feature Key

1. All Purpose Medical Computer

2. Portable Crew Restraint Board

3. Robotic Surgical Arm

4. Instrument Sterilization Unit

5. Convenience/Minor Treatment Medical Pack

6. Magnetic Surgical Tool Tray

7. Emergency Medical Pack

8. Magnetic Work Surface

9. Medical Pack Storage

10. Foothold
11. Automated External Defibrillator

12. Water/Oxygen/Vacuum Delivery

13. Medical Device Storage

-X-Ray

- Robotic Surgical Arm

- Ultrasound Equipment

- Electrocardiogram Equipment

14. Payload Equipment Restraint System (PERS) H-Strap

15. Clear Wall for CPR Foot Placement

Figure 2. Three Views of the Medical Bay Concept Computer Model Rendering and Design Features

Some additional medical supplies not shown in the 3D renderings are located in the designated storage area directly across from the Medical Bay. This includes cold storage for medical use, additional monitor displays, and another location for Medical Pack storage. 
3.1 Medical Packs. Medical consumables and small equipment are organized into single-sized Cargo Transfer Bags (CTBs) called Medical Packs. Modeled after the International Space Station's (ISS) Medical Kits these Medical Packs are color coded and organized by function. The eight total types of Medical Packs are as follows:

1. Convenience Medical Pack/ Minor Treatment Pack

2. Emergency Medical Treatment Pack

3. IV and Surgical Supply Pack

4. Medical Diagnostic Pack

5. Medical Supply Pack

6. Oral Medication Pack

7. Physician Equipment Pack

8. Topical and Injectable Medication Pack

Crew can select the appropriate Medical Pack from storage below the worksurface within the Medical Bay to easily provide a specific type of care. The Emergency Medical Pack and the Convenience/ Minor Treatment Medical Pack are stored via wall mount for direct access, but other Medical Packs may be quickly interchanged with these when providing other types of care.

3.2 Computer and devices The Medical Bay contains one Medical Computer that is used to maintain crew medical records and interfaces with all devices within the Medical System. Medical devices are stored together and include an X-ray system, electrocardiogram equipment, a blood pressure monitor, and an ultrasound device. The Xray system has a small receiving plate that moves with the detected location of the handheld X-ray emitter. Additionally, a robotic surgical arm provides minimally invasive surgical assistance and an automated external defibrillator is located for direct access in the event of a cardiac emergency. In order to minimize mass and consumables needed, an instrument sterilization unit is also included in the system to safely allow tools to be reused.

3.3 Workspace A slanted worksurface was designed to maximize surface area and support the neutral body position that the human body tends towards in zero gravity. The appropriate medical pack can be mounted on the wall then opened and quickly secured to the worksurface so all necessary supplies can be directly accessed. The worksurface also includes a magnetic section to set ferrous surgical tools on, and a hook and loop section to secure other supplies. Ferrous tools can also be set on magnetic trays that can be attached and moved quickly from the wall or ceiling and directly into the instrument sterilization unit. The Payload Equipment Restraint Systems (PERS) H-Strap and Single Strap were developed by Auburn University for use on the ISS (NASA, 2008). They are used in the Medical Bay to easily restrain and access tools and supplies while providing care.

3.4 Restraint system Footholds, handholds, and a patient restraint board were included in the design to restrain both a patient and caregivers in the zero-gravity environment. A patient restraint board is located on the left wall of the Medical Bay and is the primary location for surgery to be provided. The board has straps to hold a patient in place and is attached to the wall with four 
quick- release pins. The board can be quickly removed and brought to another location in the habitat in the event of a medical emergency.

\section{Mockup}

The Medical Bay computer model design went through three iterations over the course of this project with the final iteration made into a representative physical mockup constructed within the DSHCD, shown in Figure 4. The mockup is intended to be dimensionally accurate in order to perform a HFE analysis within the space and to put the Medical Bay in the greater context of the entire habitat. Beyond that, the mockup was not intended to be completely functional as this was an early iteration of the design and had the intent to be quick and cost effective to manufacture rather than to be of high-fidelity.

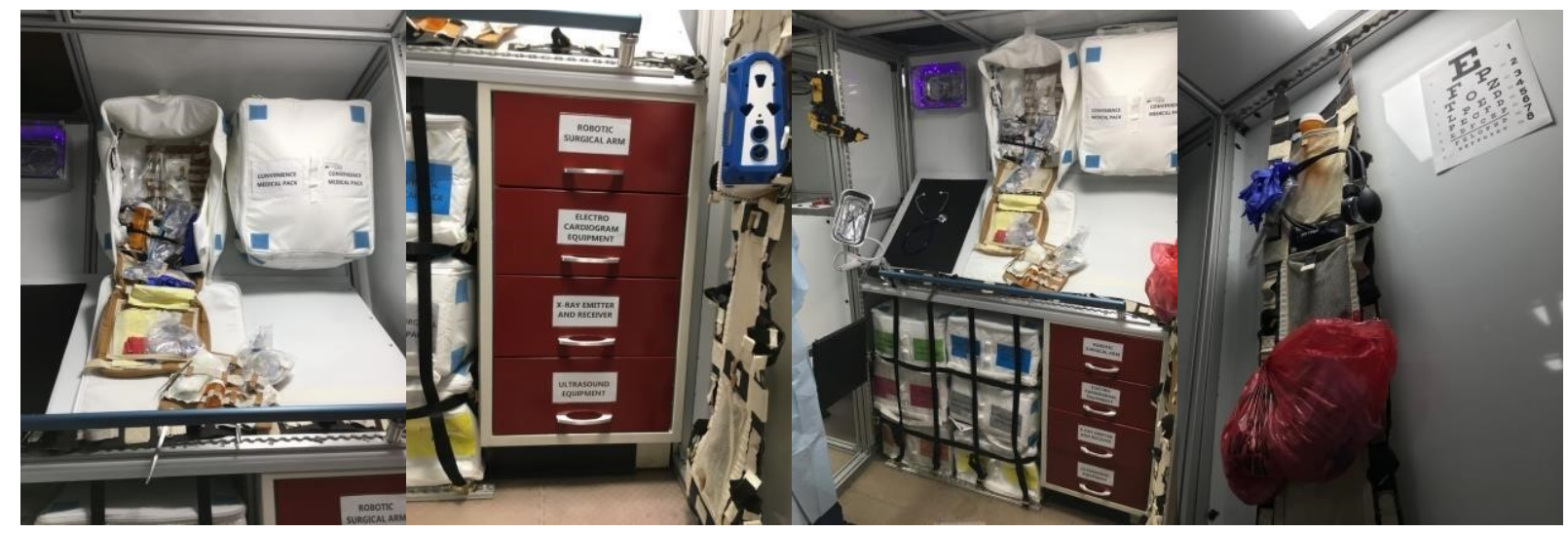

Figure 3. Medical Bay Physical Mockup in the 8.4 M Deep Space Concept Demonstrator

\section{Human Factors Engineering Analysis}

In order to assess the efficacy of the Medical Bay concept design an HFE analysis was conducted both in the physical mockup and in virtual reality. Two functions of the space were chosen to perform the initial assessment: 1) Emergency treatment of severe bleeding and 2) Emergency treatment of cardiac arrest. A task analysis was then conducted and both functions were further broken down into a collection of individual specific tasks. Each task was matched to applicable system requirements and participants were brought in to attempt to perform the tasks and meet the requirements.

In the physical mockup participants on the extremes of the body height requirements were given verbal instructions and attempted to perform each task related to emergency treatment of severe bleeding and

cardiac arrest. Throughout the test, participants, like the one shown in Figure 5, were required to secure at least one hand or foot at all times

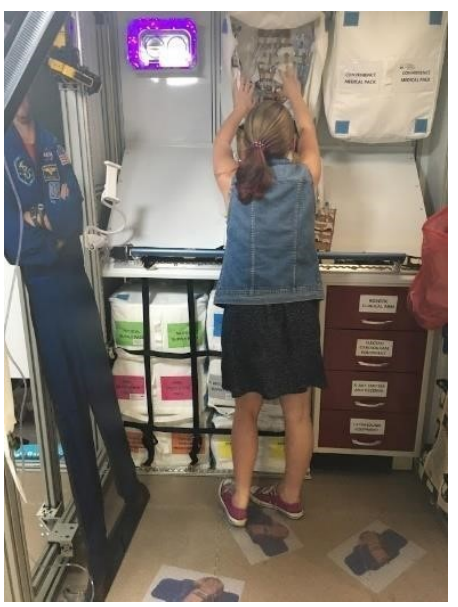

Figure 4. A 5th percentile sized female attempts to reach contents of the Emergency Medical Pack. to simulate remaining stationary in a zero-gravity environment. 
They performed as both a patient and a medical care provider for the two medical scenarios, totaling four different uses of the space tested. Observations were recorded during the test and participant feedback was gathered to determine if each task could be performed to system requirements.

A computer program, Process Simulate Human (PSH), was used to conduct additional HFE analysis in virtual reality. The 3D virtual model design was put in the program which allows the user to place human mannequins of varying sizes within the design. The mannequins can be moved in a realistic manner in order to determine if system requirements such as reach and range of motion are satisfied.

The initial Medical Bay design was shown to be a viable concept from a HFE standpoint. All tasks were able to be successfully completed by the participants in the mockup and the mannequins in PSH were able to be correctly positioned. The HFE analysis also lead to design improvements. While standing on the floor, participants close to the $1^{\text {st }}$ percentile height had some difficulty reaching supplies placed close to the ceiling. In zero gravity this would not be a concern because a person would be able to float higher to access any area of the Medical Bay. Handholds on the ceiling were added to the design so a person could support themselves while doing this. In order to maximize usable workspace the gas delivery panel was moved from the worksurface to the ceiling behind the patient restraint board and floor foot restraints were also repositioned to maximize mobility for the entire height range.

\section{Future Work}

This initial concept will continue to be used by the HFE team and ACO for further concept demonstration, analysis, and design conception for deep space travel. Since initial HFE analysis showed the Medical Bay concept layout to be a potential viable design, more analysis and design work should be completed to develop the concept to a higher-fidelity. Further research must be performed to better anticipate what health risks crew may encounter on long duration deep space travel, and as new medical technologies are developed, the Medical Bay design must be updated accordingly.

\section{Acknowledgements}

I would like to thank my mentor, Tanya Andrews, for the opportunity to work on this project and for providing leadership and invaluable advice throughout the process. Thank you also to Paul Carothers for his mockup construction help and all MSFC HFE for sharing their expertise and for

their many contributions to this project. Thank you to the Wisconsin Space Grant Consortium for their funding and the University of Wisconsin-Madison for supporting this work.

\section{References}

Dunbar, Brian. "Payload Equipment Restraint System (PERS) Fact Sheet (03/01)." NASA, NASA, 12 Apr. 2008, www.nasa.gov/centers/marshall/news/background/facts/pers.html.

Mohon, Lee. "Space Launch System (SLS) Overview.” NASA, NASA, 21 Nov. 2019, www.nasa.gov/exploration/systems/sls/overview.html. 
NASA-STD-3001 Space Flight Human-System Standard, 1, 2 NASA-STD-3001 Space Flight Human-System Standard (2015).

The Anthropometric Survey of US Army Personnel. (1988). 